\title{
DISSIPATIVE BOUSSINESQ SYSTEM OF EQUATIONS IN THE BÉNARD-MARANGONI PHENOMENON
}

\author{
R. A. Kraenkel, S. M. Kurcbart, J. G. Pereira \\ Instituto de Física Teórica \\ Universidade Estadual Paulista \\ Rua Pamplona 145, \\ 01405-900 São Paulo SP - Brazil \\ M. A. Manna \\ Laboratoire de Physique Mathématique \\ Université de Montpellier II \\ 34095 Montpellier Cedex 05 - France
}

(October 26, 2018)

\begin{abstract}
By using the long-wave approximation, a system of coupled evolution equations for the bulk velocity and the surface perturbations of a BénardMarangoni system is obtained. It includes nonlinearity, dispersion and dissipation, and it can be interpreted as a dissipative generalization of the usual Boussinesq system of equations. As a particular case, a strictly dissipative version of the Boussinesq system is obtained. Finally, some speculations are made on the nature of the physical phenomena described by this system of equations.
\end{abstract}

PACS: 47.20.Bp ; 47.35.+i

Typeset using REVTEX 


\section{INTRODUCTION}

Several recent works [1 8] have dealt with the study of oscillatory instabilities in systems of the Rayleigh-Bénard and Bénard-Marangoni type. Considering a shallow fluid layer bounded below by a plane stress-free perfect conducting plate, and with a deformable upper free surface where a constant heat flux is imposed, the linear stability analysis indicates that oscillatory motion sets in when a certain critical combination of the Rayleigh and Marangoni numbers is attained [1]. Subsequently, it was shown that, when the surface-tension can be disregarded and the Rayleigh number of the system is at $R=30$, appropriate surface deflections governed by the Korteweg-de Vries (KdV) equation appear [2]. For a two-dimensional surface, the same deflections turn out to be governed by the Kadomtsev-Petviashvili (KP) equation [3]. Thereafter, these results have been extended to the Bénard-Marangoni system [4, [], where buoyancy is discarded. In this case, appropriate surface disturbances propagate according to the $\mathrm{KdV}$ equation when the Marangoni number of the system is at $M=-12$. Further extentions to a double-diffusive systems [6], and to a temperature depending viscosity [7] have also been established. The physical mechanism behind such sustained excitations is the balance, at the critical point, between the energy dissipated by viscous forces and that released either by buoyancy, or by a temperature depending surface tension. In both cases, out of the critical points, appropriate surface excitations are governed by the Burgers equation [5, 8].

The KdV equation is well known to govern long surface-waves in inviscid shallow fluids [9]. It corresponds to a situation where nonlinearity and dispersion compensate each other, making possible the existence of coherent wave-structures, like the solitary-wave. The reductive perturbation method of Taniuti [10], based on the concept of stretching, and in which waves propagating in only one direction are sought from the beginning, is a common approach to study long waves in shallow water. From the various possible stretchings of the coordinates, different evolution equations (linear wave, breaking wave, KdV) may emerge as governing surface disturbances. However, there is an alternative approach to the 
theory of long waves in shallow water, which is based on perturbative expansions in two small parameters [9]. One of them measures the smallness of the amplitude perturbation, and the other is a measure of the longness of the wave-length perturbation. This approach, as an intermediate step, and from different possible relations between the two perturbative parameters, yields different systems of evolution equations (nonlinear shallow water, Boussinesq) describing superimposed waves propagating in opposite directions. Only when specializing to waves moving in a given direction, equations like breaking wave and $\mathrm{KdV}$ show up. A perturbative scheme of this kind has not been used to study surface excitations in Bénard systems. Consequently, for these systems, the more general evolution equations, wherefrom Burgers and KdV equations are obtained, have not been found.

In this paper, instead of using the reductive perturbation method of Taniuti [10], we will proceed through a perturbation scheme for the Bénard-Marangoni system based on two perturbative parameters, leading to the so called long-waves in shallow water approximation. By this way, a new system of coupled evolution equations will be found, involving the fluid velocity and the free-surface displacement. This system may be interpreted as a dissipative generalization of the Boussinesq equations. When the Marangoni number assumes the critical value $M=-12$, and a certain relation between the perturbative parameters is assumed, it reduces, as we are going to see, to the usual Boussinesq equations. A further restriction to waves moving in only one direction leads to the $\mathrm{KdV}$ equation. On the other hand, out of the critical point, and assuming a different relation between the perturbative parameters, the dissipative generalization of the Boussinesq equations reduce to a strictly dissipative version of the Boussinesq equations. In this case, a restriction to waves moving in only one direction will lead to the Burgers equation. Although we have restricted ourselves to the Bénard-Marangoni system, an extension to the Rayleigh-Bénard case, where buoyancy is predominant, may also be established with similar results [11. Finally, through a linear analysis, a speculation on the nature of the physical solutions to the strictly dissipative Boussinesq system of equations is made. 


\section{BASIC EQUATIONS AND BOUNDARY CONDITIONS}

We consider a fluid bounded below by a plane, stress-free, perfect termally conducting plate at $z=0$, and above by a deformable one-dimensional free surface which, at rest, lies at $z=d$. The depth $d$ will be supposed to be small enough so that buoyancy can be neglected when compared to the effects coming from the surface tension dependence on temperature. In other words, we will be dealing with a Bénard-Marangoni system. The equations that describe such a system are

$$
\begin{gathered}
\vec{\nabla} \cdot \vec{v}=0, \\
\rho \frac{d \vec{v}}{d t}=-\vec{\nabla} p+\mu \nabla^{2} \vec{v}+\vec{g} \rho, \\
\frac{d T}{d t}=\kappa \nabla^{2} T,
\end{gathered}
$$

where $\frac{d}{d t}=\frac{\partial}{\partial t}+\vec{v} \cdot \vec{\nabla}$ is the convective derivative, $\vec{v}=(u, 0, w)$ is the fluid velocity, and $p$ is the pressure. The density $\rho$, the viscosity $\mu$, and the thermal diffusivity coefficient $\kappa$ are supposed to be constant. The surface tension $\tau$ will be assumed to depend linearly on the temperature:

$$
\tau=\tau_{0}\left[1-\gamma\left(T-T_{0}\right)\right]
$$

where $\gamma$ is a constant, and $\tau_{0}, T_{0}$ are reference values for the surface tension and the temperature, respectively.

The boundary conditions on the upper free surface $z=d+\eta(x, t)$ are 12

$$
\begin{gathered}
\eta_{t}+u \eta_{x}=w, \\
\left(p-p_{a}\right)-\frac{2 \mu}{N^{2}}\left(w_{z}+u_{x} \eta_{x}^{2}-\eta_{x} u_{z}-\eta_{x} w_{x}\right)=-\frac{\tau}{N^{3}} \eta_{x x}, \\
\mu\left(1-\eta_{x}^{2}\right)\left(u_{z}+w_{x}\right)+2 \mu \eta_{x}\left(w_{z}-u_{x}\right)=N\left(\tau_{x}+\eta_{x} \tau_{z}\right),
\end{gathered}
$$




$$
\eta_{x} T_{x}-T_{z}=\frac{F}{k} N
$$

where $F$ is the normal heat flux, $k$ is the thermal conductivity, $p_{a}$ is a pressure exerted on the upper surface, all of them supposed to be constant, and $N=\left(1+\eta_{x}^{2}\right)^{\frac{1}{2}}$. Subscripts denote partial derivatives with respect to the corresponding coordinate.

On the lower plane $z=0$, we assume that the sliding resistance between two portions of the fluid is much greater than between the fluid and the plane [13], implying a stress-free lower surface:

$$
w=u_{z}=0
$$

Moreover, we will assume that the lower plate is at a constant temperature $T=T_{b}$.

\section{PERTURBATIVE SOLUTION: EVOLUTION EQUATIONS}

The static solution to the above equations is given by

$$
\begin{aligned}
& p_{s}=p_{a}-\rho g(z-d), \\
& T_{s}=T_{0}-\frac{F}{k}(z-d) .
\end{aligned}
$$

We consider now perturbations from this quiescent state. The horizontal and vertical length scales of these perturbations are supposed to be $l$ and $a$, respectively. Then, we define two small parameters

$$
\epsilon=\frac{a}{d} \quad \delta=\frac{d}{l}
$$

which will be used to order the expansions. Before proceeding further, however, it is convenient to write the equations, boundary conditions and static solutions in a dimensionless form. This is done by taking the original variables (primed) to be

$$
\begin{aligned}
& x^{\prime}=l x \quad z^{\prime}=d z \quad t^{\prime}=\frac{l}{c_{0}} t \\
& \eta^{\prime}=a \eta \quad u^{\prime}=\frac{a g}{c_{0}} u \quad w^{\prime}=\frac{a g}{c_{0} \delta} w,
\end{aligned}
$$


where $c_{0}^{2}=g d$. Furthermore, four dimensionless parameters appear: the Pradtl number $\sigma=\mu / \rho \kappa$; the Reinolds number $R=c_{0} d \rho / \mu$; the Bond number $B=\rho g d^{2} / \tau_{0}$, and the Marangoni number $M=\gamma F d^{2} \tau_{0} / k \kappa \mu$.

To obtain the nonlinear evolution of the surface perturbations in the shallow water theory, we expand all variables in powers of $z$, keeping the terms that will contribute to the evolution equations up to orders $\epsilon$ and $\delta^{2}$. Despite laborious, this procedure is straightforward, and for this reason we will omit the details, specifying in the text only the general guidelines, and giving a brief summary of the results in the Appendix. To start with, we make the expansions

$$
u=\sum_{n=0}^{\infty} z^{n} u_{n} \quad w=\sum_{n=0}^{\infty} z^{n} w_{n},
$$

where $u_{n}$ and $w_{n}$ are both functions of $x$ and $t$. Then, substituting them in Eq.(1), we get the relation

$$
w_{n+1}=-\delta^{2} \frac{u_{n x}}{n+1}
$$

Using the boundary conditions at $z=0$, it is easy to show that

$$
\begin{gathered}
w_{0}=w_{2}=w_{4}=\ldots=0, \\
u_{1}=u_{3}=u_{5}=\ldots=0 .
\end{gathered}
$$

Then, using the expansion

$$
p=p_{s}+\sum_{n=0}^{\infty} z^{n} p_{n}
$$

in Eq. (2), it is possible to obtain $u_{2}, u_{4}, u_{6}$ and $p_{2}$ in terms of $u_{0}$ and $p_{0}$ only. The other components of the expansions will contribute to orders higher than $\epsilon$ and $\delta^{2}$, and therefore they can be neglected.

Next, expanding the temperature according to

$$
T=T_{s}+\sum_{n=0}^{\infty} z^{n} \theta_{n}
$$

and using Eq. (3) with the corresponding boundary conditions, we can see that

$$
\theta_{0}=\theta_{2}=\theta_{4}=\theta_{6}=\ldots=0
$$


Moreover, we can also obtain expressions for $\theta_{3}$ and $\theta_{5}$ in terms of $u_{0}$ and $p_{0}$ only. Now, Eq.(6) yields $p_{0}$ in terms of $u_{0}$ and $\eta$. Consequently, it is possible to rewrite the $u$ 's, $p$ 's and $\theta$ 's in terms of $u_{0}$ and $\eta$ only. Finally, using the above results in Eqs. (5) and (7), we obtain, up to order $\epsilon$ and $\delta^{2}$, a coupled system of evolution equations for $u_{0}$ and $\eta$ :

$$
\begin{gathered}
u_{0 t}+\epsilon u_{0} u_{0 x}+c^{2} \eta_{x}-\frac{\delta}{R}\left(4+\frac{M}{3}\right) u_{0 x x}+\frac{\delta R}{6}\left(u_{0 t t}+\eta_{x t}\right)+\frac{\delta^{2} R^{2}}{120}\left(u_{0 t t t}+\eta_{x t t}\right) \\
-\delta^{2}\left[\frac{11}{6}-\frac{M}{30}(4 \sigma-1)\right] u_{0 x x t}-\delta^{2}\left(\frac{1}{B}+\frac{M}{30}+1\right) \eta_{x x x}=0 \\
\eta_{t}+u_{0 x}+\epsilon\left(u_{0} \eta\right)_{x}+\frac{\delta R}{6}\left(u_{0 x t}+\eta_{x x}\right)-\frac{\delta^{2}}{2} u_{0 x x x}+\frac{\delta^{2} R^{2}}{120}\left(u_{0 x t t}+\eta_{x x t}\right)=0,
\end{gathered}
$$

where

$$
c^{2}=1-\frac{M}{\sigma R^{2}}
$$

The velocity $u_{0}$ is only the first term in the expansion of $u$, which is :

$$
u=u_{0}+\delta \frac{z^{2} R^{2}}{2}\left(u_{0 t}+\eta_{x}-\frac{3 \delta}{R} u_{0 x x}\right)+\delta^{2} \frac{z^{4} R^{2}}{24}\left(u_{0 t t}+\eta_{x t}\right)+\mathcal{O}\left(\epsilon \delta, \delta^{3}\right) .
$$

The value averaged over the depth is

$$
\tilde{u}=u_{0}+\delta \frac{R}{6}\left(u_{0 t}+\eta_{x}\right)-\frac{\delta^{2}}{2}\left[u_{0 x x}-\frac{R^{2}}{60}\left(u_{0 t t}+\eta_{x t}\right)\right]+\mathcal{O}\left(\epsilon \delta, \delta^{3}\right) .
$$

The inverse is

$$
u_{0}=\tilde{u}-\delta \frac{R}{6}\left(\eta_{x}+\tilde{u}_{t}\right)+\frac{\delta^{2}}{2}\left[\tilde{u}_{x x}+\frac{7 R^{2}}{180}\left(\eta_{x t}+\tilde{u}_{t t}\right)\right]+\mathcal{O}\left(\epsilon \delta, \delta^{3}\right) .
$$

Substituting this in Eqs. (12) and (13), and using the lowest order equations into the $\tilde{u}_{x x t}$ term, we obtain (omitting the tilde):

$$
\begin{aligned}
& u_{t}+c^{2} \eta_{x}+\epsilon u u_{x}-\frac{\delta}{R}\left(4+\frac{M}{3}\right) u_{x x}+\delta^{2} \Lambda \eta_{x t t}=0 \\
& \eta_{t}+u_{x}+\epsilon(u \eta)_{x}=0
\end{aligned}
$$

where

$$
\Lambda=\frac{4}{3}-\frac{1}{c^{2}}\left(1+\frac{1}{B}\right)+\frac{M}{30}\left(1-\frac{1}{c^{2}}-4 \sigma\right)+\frac{1}{6}\left(4+\frac{M}{3}\right)\left(1-\frac{1}{c^{2}}\right)
$$


Due to the presence of the $u_{x x}$ term in Eqs. (15), this system can be considered as a dissipative generalization of the Boussinesq equations. When the Marangoni number is at the critical value $M=-12$, these equations coincide with the usual Boussinesq system of equations

$$
\begin{aligned}
& u_{t}+c^{2} \eta_{x}+\epsilon u u_{x}+\delta^{2} \Lambda \eta_{x t t}=0 \\
& \eta_{t}+u_{x}+\epsilon(u \eta)_{x}=0
\end{aligned}
$$

In this case, by assuming that $\delta^{2} \approx \epsilon$, and by specializing to waves moving, say, to the right, we can obtain a relation between $u$ and $\eta$ :

$$
u=c \eta-\epsilon \frac{c}{4} \eta^{2}-\delta^{2} \frac{\Lambda}{2} \eta_{x t}
$$

which is a kind of Riemann invariant [9]. Using this in Eq. (15) yields

$$
\eta_{t}+c \eta_{x}+\epsilon \frac{3 c}{2} \eta \eta_{x}+\delta^{2} \frac{\Lambda}{2} \eta_{x x x}=0
$$

where now

$$
\Lambda=\frac{1}{5}\left(\frac{14}{3}+8 \sigma\right)-\frac{1}{c^{2}}\left(\frac{3}{5}+\frac{1}{B}\right)
$$

Equation(19) is the KdV equation.

Let us now consider the case $M \neq-12$. Assuming that $\delta \approx \epsilon$, and neglecting terms of order $\delta^{2} \approx \epsilon^{2}$, Eq. (15) becomes:

$$
\begin{aligned}
& u_{t}+c^{2} \eta_{x}+\epsilon u u_{x}-\frac{\delta}{R}\left(4+\frac{M}{3}\right) u_{x x}=0 \\
& \eta_{t}+u_{x}+\epsilon(u \eta)_{x}=0
\end{aligned}
$$

This is a strictly dissipative version of the Boussinesq system, since the dispersive term is not present now. By specializing again to waves moving to the right, we obtain the following relation between $u$ and $\eta$ :

$$
u=c \eta-\frac{\epsilon c}{4} \eta^{2}-\frac{\delta}{2 R}\left(4+\frac{M}{3}\right) \eta_{x}
$$


Substituting this relation in Eq. (21), we obtain

$$
\eta_{t}+c \eta_{x}+\epsilon \frac{3 c}{2} \eta \eta_{x}-\frac{\delta}{2 R}\left(4+\frac{M}{3}\right) \eta_{x x}=0
$$

which is the Burgers equation.

\section{THE STRICTLY DISSIPATIVE BOUSSINESQ EQUATION}

The usual Boussinesq system of equations, Eqs. (17), may be transformed into the classical Boussinesq equations, also known as dispersive long-wave equations [14]. These equations have already been shown to be integrable [15]. The solutions to KdV and Burgers equations have also been extensively discussed in the literature [16]. However, the strictly dissipative Boussinesq system of equations, Eqs. (21), seems not to have been handled. The existence of analytical solutions, therefore, is still an open issue.

The purpose of this section is to make some speculations on the nature of the physical phenomena governed by the strictly dissipative Boussinesq system of equations. To start with, we first rewrite Eqs. (21) in a dimensional form:

$$
\begin{aligned}
& u_{t}+c^{2} g \eta_{x}+u u_{x}-\alpha d c_{0} u_{x x}=0 \\
& \eta_{t}+d u_{x}+u_{x} \eta+u \eta_{x}=0
\end{aligned}
$$

with

$$
\alpha=\frac{1}{R}\left(4+\frac{M}{3}\right)
$$

and $c^{2}$ given by Eq. (14). For $M=-12$, and changing the origin of the coordinates according to

$$
\eta(x, t)=h(x, t)-d,
$$

we obtain

$$
\begin{array}{r}
u_{t}+c^{2} g h_{x}+u u_{x}=0 \\
h_{t}+u_{x} h+u h_{x}=0
\end{array}
$$


which, when $c^{2}=1$, coincides with the classical one-dimensional shallow water system of equations [9]. A general analytical solution to this system, which might present shocks, has already been reported in the literature [17]. In fact, by restricting to waves moving to the right we obtain the breaking-wave equation [9]

$$
h_{t}-\frac{c}{2} \sqrt{g d} h_{x}+\frac{3 c}{2} \sqrt{\frac{g}{d}} h h_{x}=0 .
$$

On the other hand, for $M \neq-12$, it is possible to gain some insight on the nature of the solutions to Eqs. (24) by making a linear analysis. Neglecting the nonlinear term, Eqs. (24) reads:

$$
\begin{aligned}
& u_{t}+c^{2} g \eta_{x}-\alpha d c_{0} u_{x x}=0 \\
& \eta_{t}+d u_{x}=0 .
\end{aligned}
$$

Supposing solutions of the form $\exp (\kappa x+\omega t)$, we obtain the following dispersion relation:

$$
\omega=i \frac{\alpha d c_{0} \kappa^{2}}{2} \pm \frac{\alpha d c_{0} \kappa^{2}}{2}\left[-1+\frac{4 c^{2}}{\alpha^{2} d^{2} \kappa^{2}}\right]^{1 / 2} .
$$

There are three cases to be considered:

(i) When $4 c^{2} / \alpha^{2} d^{2} \kappa^{2}<1$, then

$$
\kappa>\frac{2 c}{\alpha d} \quad \text { or } \quad \kappa<-\frac{2 c}{\alpha d},
$$

and the frequency $\omega$ is a pure imaginary number. In this case the system will present either, a strictly dissipative or antidissipative behavior, depending on whether the imaginary part of $\omega$ is negative or positive.

(ii) When $4 c^{2} / \alpha^{2} d^{2} \kappa^{2}>1$, then

$$
-\frac{2 c}{\alpha d}<\kappa<\frac{2 c}{\alpha d},
$$

and the frequency $\omega$ is a complex number. In this case the system will present oscillatory motion combined with either dissipation or anti-dissipation, depending on whether the imaginary part of $\omega$ is negative or positive. For $\kappa$ within this interval, the nonlinear dissipative Boussinesq system, Eqs. (24), may possibly present a constant-profile opposite two 
travelling-wave solutions if a compensation mechanism between dissipation and nonlinearity takes place, in a way similar to what happens to the Taylor shock profile solution of Burgers equation [9]. This, however, is still an open question.

(iii) When $4 c^{2} / \alpha^{2} d^{2} \kappa^{2}=1$, then

$$
\kappa= \pm \frac{2 c}{\alpha d}
$$

and the frequency is again a pure imaginary number, $\omega=i \omega_{I}$, with

$$
\omega_{I}=\frac{1}{2} \alpha d c_{0} \kappa^{2}
$$

This is a transition case between the two cases previously described, in which the system will again present either a strictly dissipative or anti-dissipative behavior, depending on whether $\omega_{I}$ is negative or positive. For these values of $\kappa$, as well as for the values of case (i), the nonlinear dissipative Boussinesq system will present no oscillatory motion, which means that its solutions must describe pure nonlinear diffusive phenomena at this region.

\section{FINAL COMMENTS}

The results obtained in this paper are two-fold. First, from a perturbative analysis, corresponding to the long-wave in shallow-water approximation, we obtained a dissipative generalization of the Boussinesq system of equations as governing the bulk velocity and surface perturbations of a Bénard-Marangoni phenomenon. This system of coupled evolution equations includes nonlinearity, dispersion and dissipation. The predominance of any one of them depends on the relation between $\epsilon$ and $\delta$. Three cases are of special interest. First, when $M=-12$, the dissipative term vanishes, and assuming that $\delta^{2} \approx \epsilon$, the usual Boussinesq system of equations is obtained. Second, when the Marangoni number is far enough from the critical value, that is when $M+12=\mathcal{O}(1)$, and assuming now that $\delta \approx \epsilon$, the dissipative term dominates over the dispersive one. Then, by neglecting the dispersive term we got Eq. (21), which is a strictly dissipative version of the Boussinesq system of equations. And finally, as an intermediate case, we may also consider the situation in which $M+12=\mathcal{O}(\delta)$. 
Assuming again that $\delta^{2} \approx \epsilon$, it results that the dispersive and dissipative terms of Eq. (15) are of the same order. In this case, no term is neglected, and the generalized Boussinesq system, Eq. (15), will govern the bulk velocity and surface perturbations of the BénardMarangoni system. Upon specialization to waves moving, say, to the right, each one of these three cases will lead to a single evolution equation for the surface displacement, which will be, respectively, the $\mathrm{KdV}$, Burgers and $\mathrm{KdV}$-Burgers equations. It should be remarked that the main feature of the perturbative scheme adopted here was to allow for a clear distinction of the relative importance of each term in Eq.(15). By considering different relations between the parameters $\delta$ and $\epsilon$, a criteria to collect terms of the dominant order, and to neglect higher order ones, was thus established.

The second result of this paper refers to the appearance of a new equation, which we have called the strictly dissipative Boussinesq system of equations. It seems to be a nonintegrable equation, and the existence of analytical solutions is still an open question. Despite of this problem, we have performed here a linear analysis to get some insight on the nature of the physical phenomena it might describe. Finally, due to the fact that it shows up in a physical system, we believe this system of equation deserves further investigations as only few results exist with respect to dissipative systems.

\section{ACKNOWLEDGMENTS}

The authors would like to thank Conselho Nacional de Desenvolvimento Científico e Tecnológico (CNPq), Brazil, for partial support. One of the authors (MAM) would like also to thank the Instituto de Física Teórica, UNESP, for the kind hospitality, and the CNPq for a travel grant.

\section{APPENDIX:}

We give here a summary of the results described, but not explicitly shown in the text. The expressions for each term of the velocity expansion, already written in terms of $u_{0}$ and 
$p_{0}$, and considering only those terms that will contribute to the evolution equations up to orders $\epsilon$ and $\delta^{2}$, are

$$
\begin{gathered}
u_{2}=\frac{\delta R}{2}\left(u_{0 t}+\epsilon u_{0} u_{0 x}+\frac{1}{\epsilon} p_{0 x}-\frac{\delta}{R} u_{0 x x}\right) \\
u_{4}=\frac{\delta^{3} R}{24}\left(-\frac{2}{\epsilon} p_{0 x x x}+\frac{R}{\delta} u_{0 t t}+\frac{R}{\epsilon \delta} p_{0 x t}-2 u_{0 x x t}\right), \\
u_{6}=\frac{\delta^{3} R^{3}}{720}\left(u_{0 t t t}+\frac{1}{\epsilon} p_{0 x t t}\right) .
\end{gathered}
$$

The next terms of the expansion will not contribute to the evolution equations. In the same way, the only term of the pressure expansion that will contribute is

$$
p_{2}=-\frac{\delta^{2}}{2} p_{0 x x}
$$

From Eq. (3), with the corresponding boundary conditions, we can obtain expressions for $\theta_{1}, \theta_{3}$ and $\theta_{5}$ in terms of $u_{0}$ and $p_{0}$ only:

$$
\begin{gathered}
\theta_{1}=-\epsilon \delta \frac{R \sigma}{2} u_{0 x}+\epsilon \delta^{2} \frac{R^{2} \sigma}{24}(5 \sigma-1) u_{0 x t}-\delta^{2} \frac{R^{2} \sigma}{24} p_{0 x x}, \\
\theta_{3}=-\epsilon \delta^{2} \frac{R^{2} \sigma^{2}}{12} u_{0 x t}+\epsilon \delta^{3} \frac{R^{2} \sigma^{2}}{24}(5 \sigma-1) u_{0 x t t}+\epsilon \delta \frac{R \sigma}{6} u_{0 x} \\
\theta_{5}=\epsilon \delta^{2}\left[\left(1+\frac{1}{\sigma}\right) u_{0 x t}+\frac{1}{\epsilon \sigma} p_{0 x x}-\delta \frac{R \sigma}{2} u_{0 x t t}-\delta \frac{2}{R \sigma} u_{0 x x x}\right] .
\end{gathered}
$$

Again, these are the only components of the temperature expansion that will contribute, up to orders $\epsilon$ and $\delta^{2}$, to the evolution equations.

Now, from Eq. (6), we can get $p_{0}$ in terms of $u_{0}$ and $\eta$ only:

$$
p_{0}=\epsilon \eta-\epsilon \delta \frac{2}{R} u_{0 x}-\epsilon \delta^{2}\left[u_{0 x t}+\left(\frac{1}{B}+\frac{1}{2}\right) \eta_{x x}\right]+\epsilon \delta^{3}\left[\frac{2}{R} u_{0 x x x}-\frac{R}{12} u_{0 x t t}\right]
$$

This equation allows us to rewrite the $u$ 's, $p$ 's and $\theta$ 's in terms of $u_{0}$ and $\eta$. Consequently, the system of evolution equations, Eq. (12), obtained from Eqs. (5) and (7) could also be written in terms of $u_{0}$ and $\eta$ only. 


\section{REFERENCES}

[1] R. D. Benguria and M. C. Depassier, Phys. Fluids A 1, 1123 (1989).

[2] C. M. Alfaro and M. C. Depassier, Phys. Rev. Lett. 62, 2597 (1989).

[3] S. M. Kurcbart, M. A. Manna, J. G. Pereira and A. N. Garazo, Phys. Lett. A 148, 53 (1990).

[4] A. N. Garazo and M. G. Velarde, Phys. Fluids A 3, 2295 (1991).

[5] R. A. Kraenkel, J. G. Pereira and M. A. Manna, Phys. Rev. A 46, 4786 (1992).

[6] R. A. Kraenkel, J. G. Pereira and M. A. Manna, Physica Scripta 45, 289 (1992).

[7] R. A. Kraenkel, S. M. Kurcbart, J. G. Pereira and M. A. Manna, Phys. Lett. A 169, 259 (1992).

[8] R. A. Kraenkel, J. G. Pereira and M. A. Manna, Phys. Rev. A 45, 838 (1992).

[9] G. B. Whitham, Linear and Nonlinear Waves (Wiley, New York, 1974).

[10] T. Taniuti, Suppl. Prog. Theor. Phys. 55, 1 (1974).

[11] R. A. Kraenkel, S. M. Kurcbart, J. G. Pereira and M. A. Manna, (in preparation).

[12] J. V. Wehausen and E. V. Laitone, in Encyclopaedia of Physics, Vol. 9, edited by S. Flügge (Springer, Berlin, 1960).

[13] G. G. Stokes, Trans. Cambridge Philos. Soc. 8, 287 (1845) [reprinted in Mathematical and physical papers, Vol. 1 (Johnson Reprint Corporation, New York, 1966) pg. 75].

[14] L. F. J. Broer, Appl. Sci. Res. 31, 377 (1975).

[15] D. J. Kaup, Prog. Theor. Phys. 54, 396 (1975).

[16] R. K. Dodd, J. C. Eilbeck, J. D. Gibbon and H. C. Morris, Solitons and Nonlinear Wave Equations (Academic Press, London, 1982). 
[17] E. A. Novikov, Phys. Lett. A 123, 287 (1987). 